\title{
Morfofisiologia de plantas de couve manteiga sob concentrações de húmus líquido
}

\author{
Morphophysiology of plants of butter collard as function of liquid humus concentration \\ Maria Eunice Lima Rocha*, Pablo Wenderson Ribeiro Coutinho, Mayra Taniely Ribeiro Abade, \\ Adriano Mitio Inagaki, Danielle Acco Cadorin e Livia Maria Lemos Hoepers
}

Universidade Estadual do Oeste do Paraná, Marechal Cândido Rondon, PR, Brasil. *Autor para correspondência:

eunice_agronomia@yahoo.com.br.

Submissão: 20/05/2017 / Aceite: 01/07/2019

\begin{abstract}
RESUMO
A couve manteiga (Brassica oleracea var. acephala L.) pertence à família Brassicaceae e vários estudos têm demonstrado as variações ocorridas nas trocas gasosas entre as plantas e a atmosfera, em virtude de práticas ou de tratamentos aplicados às culturas agrícolas, com consequentes estados de estresse e reflexos negativos sobre os processos metabólicos das plantas. Além de ser uma opção para quem busca produção orgânica, o húmus líquido é uma estratégia econômica e eficiente que com inúmeros benefícios, tanto econômicos quanto agronômicos. Neste sentido, objetivou-se, avaliar algumas variáveis fisiológicas e morfológicas em plantas de couve, com concentrações de húmus líquido. O experimento foi conduzido em ambiente protegido na área experimental do setor de Horticultura e Controle Biológico pertencente à UNIOESTE, no município de Marechal Cândido Rondon, PR. Os tratamentos foram constituídos por cinco doses de húmus líquido $(0,5,10,15$ e 20\%). A adubação com o húmus líquido influenciou significativamente a fotossíntese líquida, concentração interna de $\mathrm{CO}_{2}$ e eficiência do uso da água. Massas secas de caule, folha e total não foram influenciadas pelos tratamentos. O número de folhas por planta e a área foliar foram afetadas pelas concentrações de húmus líquido. Apesar das variáveis transpiração e condutância estomática não terem apresentado diferença significativa, os demais parâmetros apresentaram resultados positivos em relação ao acréscimo de húmus líquido. Entretanto, o maior número de folhas por planta não influenciou na taxa de assimilação de $\mathrm{CO}_{2}$.
\end{abstract}

PALAVRAS-CHAVE: adubação orgânica, Brassica oleracea, fotossíntese líquida.

\begin{abstract}
Brassica cabbage (Brassica oleracea var. Acephala L.) belongs to the Brassicaceae family and several studies have shown the variations in gas exchange between plants and the atmosphere, due to practices or treatments applied to agricultural crops, with consequent stress and negative reflexes on the metabolic processes of plants. In addition to being an option for those seeking organic production, liquid humus is an economic and efficient strategy with numerous benefits, both economic and agronomic. In this sense, the objective was to evaluate some physiological and morphological variables in cabbage plants, with concentrations of liquid humus. The experiment was carried out in a protected environment in the experimental area of Horticulture and Biological Control, belonging to UNIOESTE, in the city of Marechal Cândido Rondon, PR. The treatments consisted of five doses of liquid humus $(0,5,10,15$, and $20 \%)$. Fertilization with liquid humus significantly influenced liquid photosynthesis, internal $\mathrm{CO}_{2}$ concentration and water use efficiency. Dry masses of stem, leaf, and total were not influenced by the treatments. The number of leaves per plant and the leaf area were affected by the concentrations of liquid humus. Although the variables transpiration and stomatal conductance did not present significant difference, the other parameters presented positive results in relation to the increase of liquid humus. However, the higher number of leaves per plant did not influence the assimilation rate of $\mathrm{CO}_{2}$.
\end{abstract}

KEYWORDS: organic fertilization, Brassica oleracea, liquid photosynthesis.

\section{INTRODUÇÃO}

A couve manteiga (Brassica oleracea var. acephala L.) pertence à família Brassicaceae, não forma cabeça e suas folhas apresentam limbo bem desenvolvido, arredondado, com pecíolo longo e nervuras bem 
destacadas (FILGUEIRA 2013). É uma hortaliça arbustiva anual ou bienal, cujo consumo no Brasil tem aumentado gradativamente devido, provavelmente, às novas maneiras de utilização na culinária e as recentes descobertas da ciência quanto as suas propriedades nutracêuticas (NOVO et al. 2010).

É uma cultura típica de outono-inverno se desenvolvendo melhor em temperaturas mais amenas, entre 16 e $22{ }^{\circ} \mathrm{C}$, apresentando certa tolerância ao calor, podendo, em algumas regiões ser plantada ao longo de todo ano (FILGUEIRA 2013). Pode permanecer produtiva por vários meses, mas é altamente exigente em água (HUSSAR et al. 2004).

O húmus líquido apresenta-se como uma opção para a adubação orgânica em hortaliças. É uma solução obtida pela mistura de húmus sólido e água e apresenta em sua composição, nutrientes minerais e ácidos orgânicos que estimulam o crescimento e desenvolvimento das plantas. Além disso, há uma gama de microrganismos, que, por sua vez, favorecem as relações de equilíbrio entre as populações existentes no solo (ARTEAGA et al. 2007).

Devido à disponibilidade de elementos químicos essenciais no húmus, busca-se oferecer esses nutrientes de forma mais rápida às culturas por irrigação ou foliar. No caso de culturas como a couve, em que as folhas constituem a parte comercializável, evita-se o contato do fertilizante com as mesmas. A proporção do composto sólido e da água utilizada determina sua concentração. A Embrapa estima que a concentração mais adaptável à condição de fornecimento do fertilizante via foliar, seja de $15 \%$ (SCHIEDECK et al. 2008).

Para o entendimento do metabolismo da planta e avaliação do seu estresse, é necessário conhecer tanto a morfologia quanto as variáveis fisiológicas das mesmas, neste sentido é importante à quantificação de características como, pigmentos fotoprotetores de folhas, abertura estomática, fotossíntese líquida, perda de água por transpiração foliar, variáveis morfobiométricas, e outros parâmetros.

A influência do estado nutricional da planta sobre a fotossíntese e a respiração ocorre de muitas maneiras, sendo que quase sempre maiores taxas fotossintéticas são alcançadas por meio da adubação. Vários estudos demonstram as variações ocorridas nas trocas gasosas, em virtude de práticas ou de tratamentos aplicados às culturas agrícolas, com consequentes estados de estresse e reflexos sobre os processos metabólicos das plantas, inclusive com variações nos níveis de aminoácidos (CECHIN et al. 2006, PRAXEDES et al. 2006, SANTOS et al. 2006).

Além de ser uma opção para quem busca a produção orgânica, o húmus líquido é uma estratégia econômica e eficiente que pode trazer benefícios, tanto econômicos quanto agronômicos. Neste sentido, objetivou-se avaliar características morfofisiológicas e plantas de couve manteiga em função de concentrações de húmus líquido, via solo.

\section{MATERIAL E MÉTODOS}

O experimento foi conduzido em ambiente protegido na área experimental do setor de Horticultura e Controle Biológico, pertencente à UNIOESTE, no município de Marechal Cândido Rondon, PR, no período de março a junho de 2015. O clima, classificado segundo Köppen, é do tipo Cfa, subtropical, com média anual de precipitação de $1.700 \mathrm{~mm}$, mantendo-se a média anual de temperatura entre $22{ }^{\circ} \mathrm{C}$ e $23^{\circ} \mathrm{C}$ (ALVARES et al. 2013). Os valores médios, mínimo e máximo de temperatura do ar e média de umidade relativa do ar por dia durante o ciclo da couve estão descritos na Figura 1.

O delineamento experimental utilizado foi inteiramente casualizado, com quatro repetições. Os tratamentos foram constituídos por cinco concentrações de húmus líquido (0, 5, 10, 15 e 20\%), sendo preparada conforme metodologia proposta por SCHIEDECK et al. (2008), onde a análise química do húmus líquido é descrita na Tabela 1, sendo diluído conforme a concentração.

A aplicação dos tratamentos ocorreu em forma de pulverização, sendo aplicado semanalmente por vaso $0,450 \mathrm{~L}$, com um total durante o ciclo de $3,600 \mathrm{~L}$, conforme o tratamento onde a concentração $0 \%$ era aplicado apenas água.

As mudas da couve 'Geórgia' foram produzidas em bandejas de poliestireno expandido de 200 células, utilizando substrato comercial para hortaliças. Aos 28 dias após semeadura, quando apresentaram segunda folha verdadeira, procedeu-se o transplante para vasos de polietileno, com volume de $12 \mathrm{dm}^{3}$, preenchidos com substrato comercial para hortaliças e vermicomposto sólido na proporção de 1:1 (Tabela 2). O espaçamento foi de $1,20 \mathrm{~m}$ entre linhas e $0,5 \mathrm{~m}$ entre plantas.

A irrigação foi realizada via gotejamento, utilizando-se fita flexível com vazão de 1,6 $\mathrm{L} \mathrm{h}^{-1}$ e emissores espaçados a cada $0,50 \mathrm{~m}$, conforme espaçamento entre plantas, sendo realizado irrigação duas vezes ao dia para manter o substrato com umidade acima de $80 \%$, conforme a necessidade da cultura. O controle fitossanitário foi realizado de acordo com a necessidade da cultura, sendo realizadas duas aplicações de 
inseticidas aos 37 e 47 dias após o transplantio (DAT) para controle de pulgão-verde (Myzus persicae) e mosca-branca (Bemisia tabaci biótipo B).
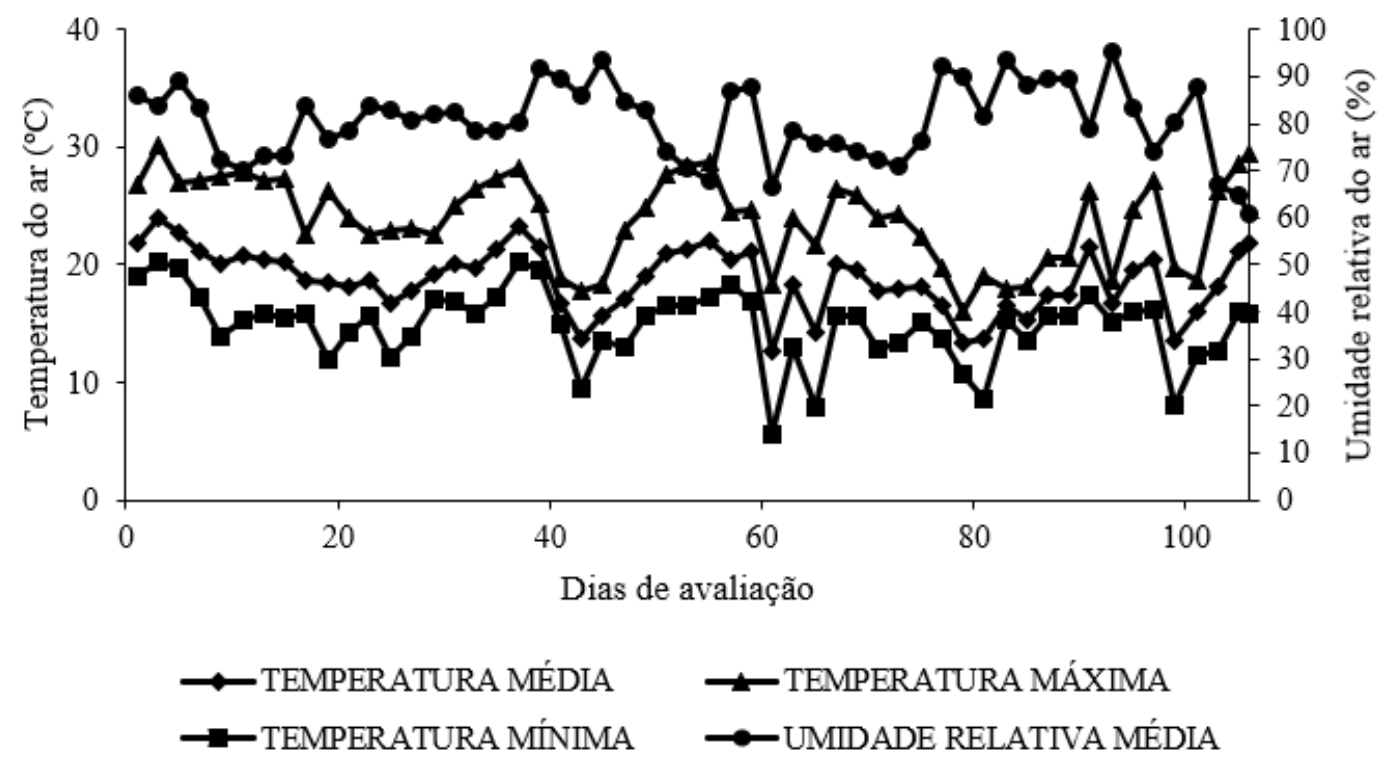

Figura 1. Valores médio, mínimo e máximo de temperatura do ar e média de umidade relativa do ar por dia, durante o ciclo da couve manteiga.

Figure 1. Mean, minimum and maximum values of air temperature and average relative humidity of air per day during the cycle collard.

Aos 61 dias após o transplantio, foram realizadas medidas de taxa de assimilação líquida de $\mathrm{CO}_{2}(\mathrm{~A})$, transpiração foliar (E), condutância estomática (gs) e concentração interna de $\mathrm{CO}_{2}(\mathrm{Ci})$. As avaliações foram realizadas na quinta folha, totalmente expandida, a partir do ápice da planta, entre as 9 e 11 horas da manhã, em um dia ensolarado e sem nebulosidade.

A intensidade média de luz ambiente observada foi de $648,78 \mu \mathrm{mols}^{-2} \mathrm{~s}^{-1}$, umidade relativa do ambiente de $51,02 \%$ e concentração de $\mathrm{CO}_{2}$ foi de $389,26 \mathrm{ppm}$. Foi utilizando um analisador portátil de fotossíntese por radiação infravermelha (Infra Red Gas Analyser - IRGA, modelo Li-6400XT, LI-COR), com fonte de luz constante de $1200 \mu \mathrm{mols} \mathrm{m}^{-2} \mathrm{~s}^{-1}$. Ainda foi calculada a eficiência no uso da água (EUA), obtido pela razão entre a taxa de assimilação líquida de $\mathrm{CO}_{2}$ e transpiração foliar (BERRY \& DOWNTON 1982).

Tabela 1. Composição química média do húmus líquido utilizado no experimento.

Table 1. Mean chemical composition of the liquid humus used in the experiment.

\begin{tabular}{ccccccccccccccc}
\hline & $\mathrm{P}$ & $\mathrm{N}$ & $\mathrm{K}$ & $\mathrm{Ca}$ & $\mathrm{Mg}$ & $\mathrm{S}$ & $\mathrm{Co}$ & $\mathrm{Mo}$ & $\mathrm{Cu}$ & $\mathrm{Zn}$ & $\mathrm{Mn}$ & $\mathrm{B}$ & $\mathrm{Fe}$ & $\mathrm{pH}$ \\
\hline $\begin{array}{c}\text { Concentrações } \\
\%\end{array}$ & 12,3 & 2,1 & 42,3 & 12,5 & 8 & 6,6 & 62,4 & 107,3 & 0,00025 & 0,00225 & 0,0005 & 0,000656 & 0,5995 & 7,22 \\
\hline
\end{tabular}

Tabela 2. Composição química da mistura utilizada no plantio das mudas.

Table 2. Chemical composition of the mixture used in planting of seedlings.

\begin{tabular}{cccccccccc}
\hline & $\mathrm{P}$ & $\mathrm{K}$ & $\mathrm{Ca}$ & $\mathrm{Mg}$ & $\mathrm{H}+\mathrm{Al}$ & $\mathrm{Cu}$ & $\mathrm{Zn}$ & $\mathrm{Mn}$ & $\mathrm{Fe}$ \\
\hline $\begin{array}{c}\text { Concentrações } \\
\%\end{array}$ & $\%$ & \multicolumn{6}{c}{$\mathrm{cmol}_{\mathrm{c}} \mathrm{dm}^{-3}$} \\
$\%$ & 47,405 & 2,97 & 10,30 & 5,64 & 4,02 & 0,44 & 0,014 & 13,658 & 0,911 \\
\hline
\end{tabular}

Aos 68 DAT, foi realizada a colheita, sendo avaliadas variáveis morfométricas, dentre elas, área foliar, determinada pelo aparelho Area Meter da marca Li-cor, modelo LI3100C, número de folhas por planta e massa seca de folha, caule e total. A massa seca total foi determinada pela soma das massas de folha e de caule, obtidas após a secagem em estufa, com circulação de ar forçada a $65{ }^{\circ} \mathrm{C}$, até atingirem peso constante. Os valores foram expressos em g por planta. 
Os dados experimentais foram submetidos aos testes de normalidade e homogeneidade de variância por meio dos testes de Lilliefors ( $p \leq 0,05)$, através do software estatístico ASSISTAT. Em seguida, procedeu à análise de variância e de regressão polinomial (BANZATTO \& KRONKA 2006) ( $\leq \leq 0,05)$, utilizando-se o software estatístico SISVAR 5.3 (FERREIRA 2014).

\section{RESULTADOS E DISCUSSÃO}

Concentrações de húmus líquido influenciaram, de forma linear, a fotossíntese líquida $(A)$, a concentração interna de $\mathrm{CO}_{2}(\mathrm{Ci})$ e a eficiência no uso da água (EUA). Porém, transpiração foliar $(\mathrm{E})$ e a condutância estomática (gs) não apresentaram significância pelo teste $F$, tanto para modelo linear quanto quadrático.

O modelo polinomial que melhor se ajustou ao comportamento da variável fotossíntese líquida, em função de concentrações de húmus líquido, foi linear (Figura 2a). Desta forma, quanto maior a concentração de húmus na solução aplicada, maior a fixação de $\mathrm{CO}_{2}$ no mesofilo foliar. O maior valor de $\mathrm{A}(22,26 \mu$ mol $\mathrm{m}^{-2} \mathrm{~s}^{-1}$ ) foi obtido com a maior concentração de húmus (20\%).

A aplicação de maior volume do fertilizante orgânico pode ter contribuído para maior disponibilidade de nutrientes às raízes e, consequentemente aumentado a produção de fotoassimilados, mas para afirmarse com total certeza seria necessário avaliar o teor foliar de nutrientes.

As taxas de fotossíntese líquida foram maiores aos valores encontrados por LACERDA et al. (2012), os quais avaliaram couve manteiga 'Geórgia' em função de substratos e concentrações de nutrientes na solução nutritiva. Uma justificativa poderia ser atribuída ao uso do substrato utilizado no trabalho, pois a mistura de húmus sólido com substrato comercial apresentou alta fertilidade, principalmente ao teor de fósforo (474,05 ppm), além de apresentar eficiência em armazenar umidade e, desta forma, a perda por lixiviação foi reduzida.

A concentração interna de $\mathrm{CO}_{2}(\mathrm{Ci})$, apresentou correlação inversamente proporcional com o aumento da concentração de húmus líquido aplicado ao solo. A redução de $\mathrm{Ci}$ se torna coerente devido à relação da abertura estomática, entrada de $\mathrm{CO}_{2} \mathrm{e}$, consequentemente, sua fixação na formação de novos carboidratos (Figura $2 b$ ).
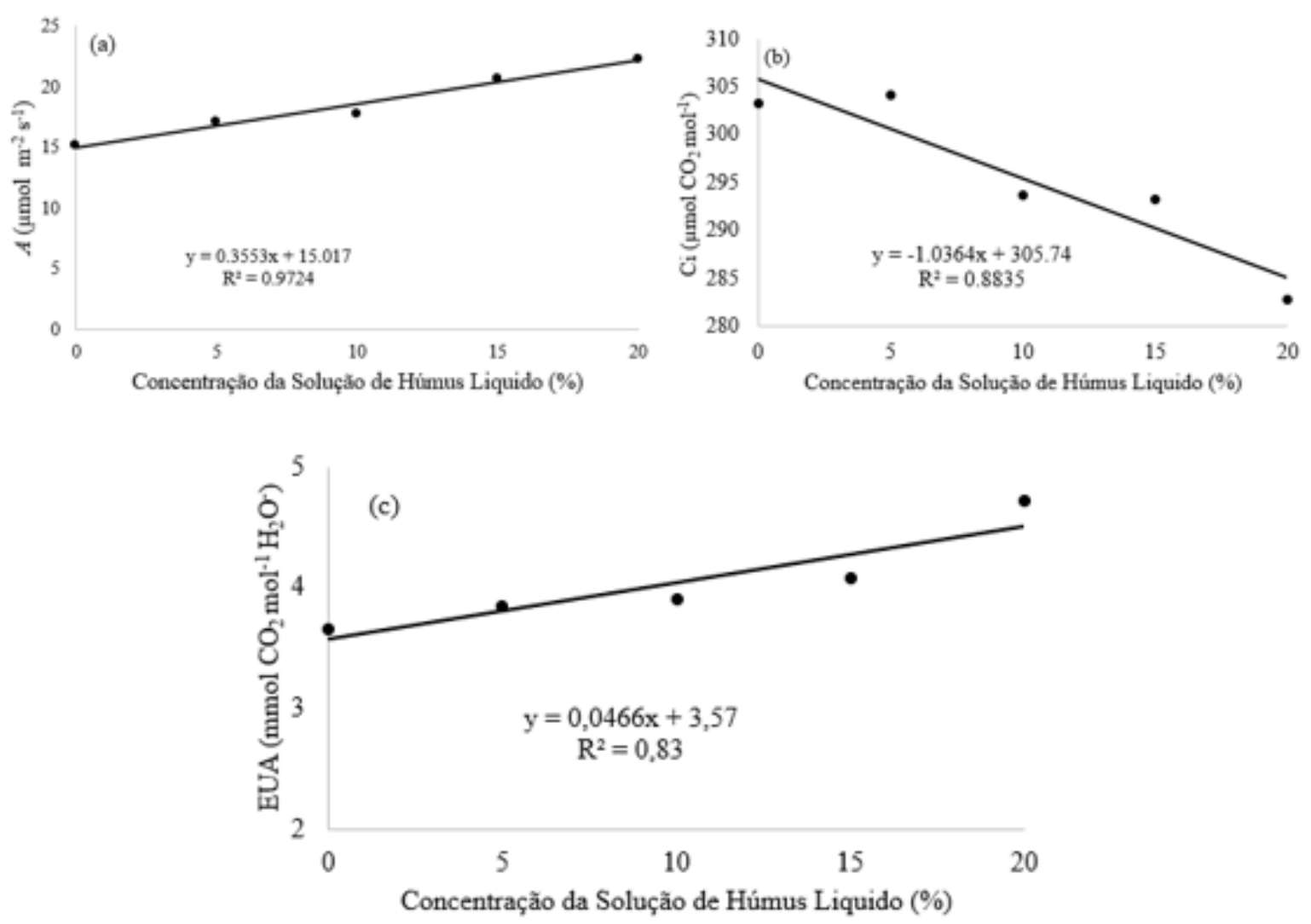

Figura 2. Taxa de assimilação líquida de $\mathrm{CO}_{2}$ (a), concentração interna de $\mathrm{CO}_{2}$ (b) e eficiência do uso da água (c) em plantas de couve manteiga 'Geórgia'

Figure 2. Net assimilation rate of $\mathrm{CO}_{2}(a)$, internal $\mathrm{CO}_{2}$ concentration (b), water use efficiency (c) of Georgia kale. 
A condutância estomática (gs) não apresentou diferença significativa, foi observado média de 0,513 mols $\mathrm{m}^{-2} \mathrm{~s}^{-1}$, o que apresenta abertura estomática suficiente para a entrada de $\mathrm{CO}_{2}$ atmosférico por difusão de um meio mais concentrado (400 ppm). Com abertura estomática semelhante para todos os tratamentos, a entrada de $\mathrm{CO}_{2}$ também deve ser semelhante (TAIZ \& ZEIGER 2013). No entanto, o valor de Ci pode variar de acordo com a eficiência de carboxilação, ou seja, a fixação de $\mathrm{CO}_{2}$ intermediada pela enzima Rubisco pode ser mais eficiente dependendo das condições submetidas às plantas, como luz, temperatura e principalmente nutricional, devido macro elementos como o nitrogênio (TAIZ \& ZEIGER 2013).

$A$ gs e $E$ foram semelhantes para todas as concentrações de húmus estudadas $(p>0,05)$. Segundo RASCHKE (1979), menores valores de Ci estimulam a abertura dos estômatos, com consequente aumento na taxa de assimilação de dióxido de carbono. Desta forma, a melhor equação que se ajustou para a eficiência no uso da água (EUA) foi a linear (Figura 2c). Conforme aumentou-se a concentração de húmus, houve aumento para EUA de forma linear, obtendo maior média em $20 \%$ de concentração da solução.

Como consequência de gs apresentar semelhança para todos os tratamentos, a perda de água por transpiração foliar também não se diferenciou conforme concentrações de húmus. Porém, com maiores concentrações de húmus, as plantas se tornaram mais nutridas e com maior capacidade de fixar carbono, no entanto, com as mesmas taxas de E.

Nas análises de variância para massa de matéria seca de caule, folha e total, não foram observados efeitos significativos das concentrações de húmus líquido. Entretanto, foram constatados para número de folhas por planta (Figura 3b) e área foliar (Figura 3a).
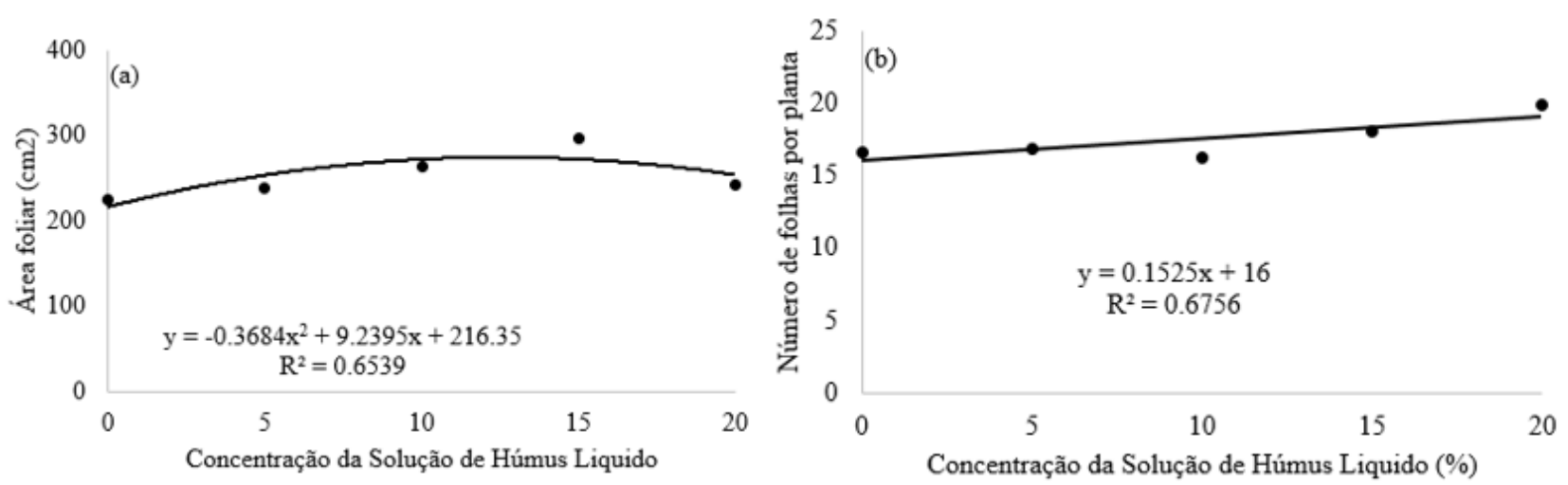

Figura 3. Área foliar (a) e número de folhas por planta (b) de couve manteiga 'Geórgia' submetido a diferentes concentrações de húmus líquido em ambiente protegido.

Figure 3. Leaf area (a) and numbers of leaves per plant (b) of Georgia kale submitted to different concentrations of liquid humus in protected environment.

Conforme equação quadrática obtida pela análise de regressão, plantas de couve atingiram maior área foliar de $274,28 \mathrm{~cm}^{2} \mathrm{com} 12,54 \%$ de húmus líquido de solução aplicado (Figura $3 a$ ).

A taxa de assimilação de $\mathrm{CO}_{2}$ foi aumentando de acordo com as concentrações de húmus líquido, mas isso não refletiu positivamente nos índices fisiológicos que compõem a análise de crescimento ao longo do ciclo, ou seja, não resultaram em aumento de matéria seca. A adubação em hortaliças folhosas favorece o desenvolvimento vegetativo elevando seu potencial produtivo da cultura (LARCHER 2000).

\section{CONCLUSÃO}

O aumento na porcentagem de húmus líquido em couve manteiga resultou em incremento na eficiência do uso de água e em contrapartida reduziu a concentração interna de $\mathrm{CO}_{2}$, isso pode ter ocorrido porque, mediante a imposição do tratamento os estômatos fecharam, reduzindo a perda de água e consequentemente reduzindo o aporte de gás carbônico, essa estratégia pode ser desvantajosa a longo prazo e por isso, não se recomenda a aplicação de doses mais elevadas de húmus, nessa condição. $O$ acréscimo da concentração de húmus líquido, aumentou a área fotossintética, mas não refletiu na produção de massa de matéria seca de plantas de couve folha, visto que a redução no acúmulo de gás carbônico refletiu também na produção de fotossintatos e distribuição da biomassa vegetal.

A aplicação de húmus líquido pode trazer vantagens ecológicas e agronômicas, através da redução do uso de fertilizantes sintéticos de alta solubilidade, ao mesmo tempo aumenta-se a fertilidade do solo de forma natural e orgânica, porém o uso deve ser feito com cautela e em doses não elevadas, visto que pode alterar as características fisiológica das plantas. 


\section{REFERÊNCIAS}

ALMEIDA LP. 2001. Germinação, crescimento inicial e anatomia foliar de plantas jovens de Cryptocarya aschersoniana Mez. sob diferentes níveis de radiação. Dissertação (Mestrado em Fisiologia Vegetal). Lavras: UFV. 96p.

ALVARES CA et al. 2013. Köppen's climate classification map for Brazil. Meteorologische Zeitschrift 22: 711-728.

ARTEAGA M et al. 2007. Influência de la aplicación foliar del bioestimulante Liplant sobre algunos indicadores biológicos del suelo. Revista de Protección Vegetal 22: 110-117.

BANZATTO DA \& KRONKA SN. 2006. Experimentação agrícola. 4.ed. Jaboticabal: Funep. 237p.

BERRY JA \& DOWNTON WJS. 1982. Environmental regulation of photosyntesis. In: GOVINDJEE, E. Photosynthesis: development, carbono metabolismo, and plant production. New York, Academic Press 2: 306-308.

CECHIN I et al. 2006. Photosynthetic responses and proline content of mature and young leaves of sunflower plants under water deficit. Photosynthetica 44: 143-146.

FERREIRA DF. 2014. Sisvar: a Guide for its Bootstrao procedures in multiple comparisons. Ciência e Agrotecnologia 38 : 109-112.

FILGUEIRA FAR. 2013. Novo manual de olericultura: Agrotecnologia moderna na produção e comercialização de hortaliças. 3.ed. Viçosa: UFV. 412p.

HUSSAR GJ et al. 2004. Efeito do uso do efluente de reator anaeróbio compartimentado na fertirrigação da couve. Revista Ecossistema 29: 65-72.

LACERDA FHD et al. 2012. Substrato e concentração de nutrientes na solução nutritiva na produção de couve manteiga. Revista Verde 7: 51-58.

LARCHER W. 2000. Ecofisiologia Vegetal. Tradução PRADO CHBA. São Carlos: Rima. 531p.

NOVO MCSS et al. 2010. Desenvolvimento e produção de genótipos de couve manteiga. Horticultura Brasileira 28: 321 325.

PRAXEDES SC et al. 2006. Effects of long-term soil drought on photosynthesis and carbohydrate metabolism in mature robusta coffe (Coffea canephora Pierre var. kouillou) leaves. Environmental an Experimental Botany 56: 267-273.

RASCHKE K. 1979. Movements using turgor mechanisms: movements of stomata. In: HAUPT W \& FEINLEIB ME. (Ed.). Encyclopedia of Plant Physiology. Berlin: Springer-Verlag. p.383-441.

SANTOS MG et al. 2006. The role of inorganic phosphate on photosynthesis recovery of common bean after a mild water deficit. Plant Science 170: 659-664.

SCHIEDECK G et al. 2008. Preparo e uso de húmus líquido: opção para adubação orgânica em hortaliças. Pelotas: EMBRAPA 4p. (Comunicado Técnico 195).

TAIZ L \& ZEIGER E. 2013. Fisiologia Vegetal. 5.ed. ArtMed: Porto Alegre. 954p. 